\title{
2702. Effects of Casimir force on multi-field coupled nonlinear vibration of orthotropic micro film
}

\author{
Xiaorui $\mathrm{Fu}^{1}$, Lizhong $\mathrm{Xu}^{2}$ \\ Mechanical Engineering Institute, Yanshan University, Qinhuangdao, China \\ ${ }^{2}$ Corresponding author \\ E-mail: ${ }^{1781908537 @ q q . c o m,{ }^{2} x l z @ y s u . e d u . c n}$
}

Received 3 February 2017; received in revised form 1 May 2017; accepted 9 May 2017 DOI https://doi.org/10.21595/jve.2017.18226

Check for updates

\begin{abstract}
In this paper, the nonlinear dynamics equations of a multi-field coupled orthotropic micro film are presented. The Krylov-Bogoliubov-Mitropolsky (KBM) method is used to solve the equations. The multi-fields coupled nonlinear resonant frequencies of a micro film are analyzed. The effects of Casimir force and nonlinear parameter on nonlinear resonant frequencies and vibration amplitudes are investigated. A resonant film is designed and produced. Based on the principle of electrostatic excitation and capacitance detection, the resonant frequency of the micro film is detected. It illustrates that the Casimir force has an important influence on the resonant micro film frequencies and should be considered with a small initial clearance.
\end{abstract}

Keywords: Casimir force, multi-field coupled, nonlinear vibration, orthotropic micro film.

\section{Introduction}

Micro-electro-mechanical systems (MEMS) mainly include micro-sensors, micro-actuators and processing circuits [1,2]. As an important branch of MEMS devices, micro-sensors have advantages such as fast responses, high sensitivity, and are suitable for mass production, etc. [3]. Among the micro-sensors, resonant sensors output frequency signals in which the distortion will not occur in the long-distance transmission, and the signal processing circuits can be simplified largely so that they are widely used in automobile, intelligent equipment, chemical industry, quality and process control, fragrance design, oenology, etc [4]. Resonant sensor resonator mainly includes two types: film or beam. The beam resonator has a higher sensitivity than a film resonator, but its output signals are weaker, and a higher precise detection circuit is required. The output signal of the film resonant sensor is strong, and the requirement to a test circuit is low, of course, its sensitivity is lower than that of beam resonators $[5,6]$.

Micro resonant sensors work in the resonant state. Physical quantities are detected by fluctuations of the resonant frequencies. So, for a micro sensor with film resonator, determining the resonant frequency and its fluctuations along with the system parameters is an important problem. For the dynamics problem of the micro film, a lot of studies have been done.

The film vibration theory was derived from the string vibration theory in the 18th century [7]. In 1993, Alonso studied the vibration characteristics for a resonator made with $\mathrm{NiCr}$ thin film micro resonance pressure sensor [8]. Fan studied the nonlinear vibration of a micro resonant pressure sensor. The multi-scale method was used to analyze the influence of the nonlinear term on the natural frequency of the sensor [9]. Federico discussed the Casimir effect and its use in nanomechanics and nanotechnology, and finished high precision measurements of the Casimir force using microelectromechanical systems technology [10]. In 2009, Reutskiy developed a new method of numerical analysis of the nonlinear film vibration. This method is based on a mathematical simulation of the physical response of the spectrum analysis system [11]. In the same year, Goncalves analyzed the geometrical and material nonlinear vibrations of hyperelastic tensioned films under small deformation and time-dependent transverse pressure by the method of Galerkin [12]. Liu carried out a theoretical analysis of the nonlinear free vibration of thin films and the forced vibrations under impact loads [13]. The dynamics of a circular film in zero gravity was studied by Sorokin, and the spectrum of natural vibrations were given [14].

$\mathrm{J}$ Weber studied thin film resonators as environmental pressure sensors and developed a model 
to evaluate their sensitivity [15]. Steffen developed a polycrystalline silicon thin film resonator with three-layer or two-layer structure, different film strain factors, and different resistance temperature coefficients [16]. Zhao fabricated an odorant biosensor based on $\mathrm{ZnO}$ film bulk acoustic resonators (FBARs) with resonant frequency of $-1.5 \mathrm{GHz}$, and demonstrated the potential of FBARs as odorant biosensors [17]. Jiang fabricated high-performance PZT thin films with a thickness of $2.6 \mu \mathrm{m}$ which are produced on a silicon substrate by the sputtering deposition process, and a Lamb wave resonator was fabricated using the highly selective reactive ion etching process [18]. Gualdino fabricated a thin-film silicon resonator by surface micromachining and studied vibration modes of micromechanical disk resonators made of hydrogenated amorphous silicon films [19]. Ren presented a novel resonant pressure sensor with an improved micromechanical double-ended tuning fork resonator packaged in dry air at the atmospheric pressure in which the fundamental frequency of the resonant pressure sensor is approximately $34.55 \mathrm{kHz}$ with a pressure sensitivity of $20.77 \mathrm{~Hz} / \mathrm{kPa}$ [20]. Hamelin studied a new method to optically trim the resonance frequency of micro-silicon resonator coated with a film germanium layer [21]. Alahnomi developed a new sensor for detecting the properties of the materials [22].

However, the resonator of a resonant sensor operates normally under multi-fields forces. Especially, as the resonator size reduces, effects of the molecule force on its resonant frequency become more significant and could not be neglected. To predict the resonant frequency and its fluctuations of the micro film resonator accurately, the multi-field coupled vibration problem under the influence of molecular force should be investigated.

In this paper, a multi-field coupled dynamics model of the orthotropic film resonator is proposed. Here, the Casimir force, the air damping force and the electrostatic force are considered. Using the model, the nonlinear vibration frequency and displacement response of the micro film resonator are investigated. Effects of the Casimir force on the nonlinear vibration frequency for the micro film resonator are determined. To illustrate the analysis, a micro film resonator is fabricated, and the nonlinear frequencies of the resonator under different initial clearances are detected. Results show that the detected resonant frequencies are in agreement with the calculative values. The results can be used in design of the resonant frequencies for a micro resonant film sensor. A flowchart of the research idea is given in Fig. 1.

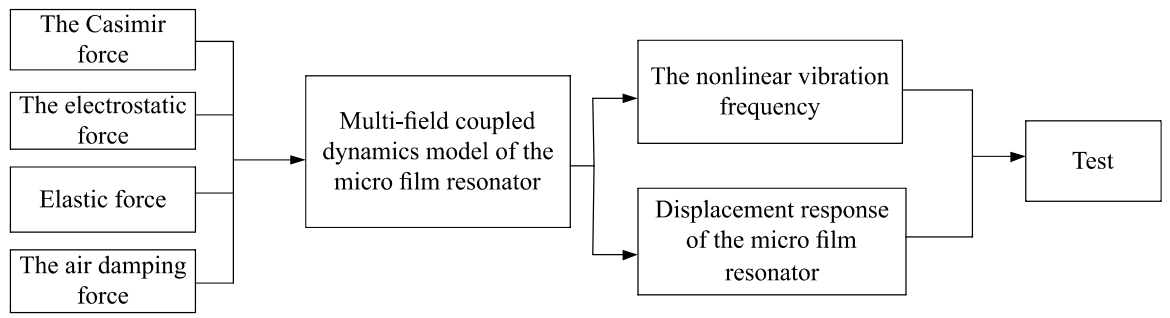

Fig. 1. Research idea flowchart

\section{Coupled dynamics equations}

An orthotropic resonant film in the micro resonant sensor is shown in Fig. 2. This is a multi-field coupled system for the micro-thin film under the action of electrostatic force, Casimir force and air damping force. Its boundary condition is that two sides are fixed and two sides are free. Here, $h$ is the film thickness, $\Delta w$ is the film vibration displacement, $a$ and $b$ are the resonant film dimensions, $v$ is the initial clearance between the film and base plate.

According to the theory of D'Alembert principle [23], the control equations of nonlinear free vibration for the film are given by:

$$
\rho h \frac{\partial^{2} \Delta w}{\partial t^{2}}+\gamma \frac{\partial \Delta w}{\partial t}-\left(\sigma_{x 0}+\frac{\partial^{2} \varphi}{\partial y^{2}}\right) h \frac{\partial^{2} \Delta w}{\partial x^{2}}-\left(\sigma_{y 0}+\frac{\partial^{2} \varphi}{\partial x^{2}}\right) h \frac{\partial^{2} \Delta w}{\partial y^{2}}=\Delta q,
$$


$\frac{1}{E_{1}} \frac{\partial^{4} \varphi}{\partial y^{4}}+\frac{1}{E_{2}} \frac{\partial^{4} \varphi}{\partial x^{4}}=\left(\frac{\partial^{2} \Delta w}{\partial x \partial y}\right)^{2}-\frac{\partial^{2} \Delta w}{\partial x^{2}} \frac{\partial^{2} \Delta w}{\partial y^{2}}$,

where $\rho$ is the density, $U_{0}$ is the voltage, $\varphi$ is the stress function, $\sigma_{x 0}$ is the initial tension stress in $x$ direction of the film, $\sigma_{y 0}$ is the initial tension stress in $y$ direction of the film, $E_{1}$ is the Young moduli in $x$ direction, $E_{2}$ is the Young moduli in $y$ direction, $\gamma$ is the damping coefficient of the air, it can be given as:

$\gamma=\frac{\mu b^{3}}{\left(v-\bar{w}_{0}\right)^{3}}$

where $\mu$ is the air dynamic viscosity, $\mu=1.86 \times 10^{-5} \mathrm{~N} \cdot \mathrm{S} \cdot \mathrm{m}^{-2}$.

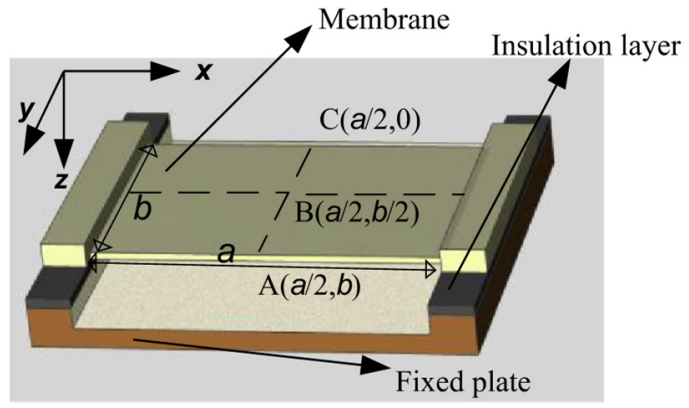

a) Structure model of micro film

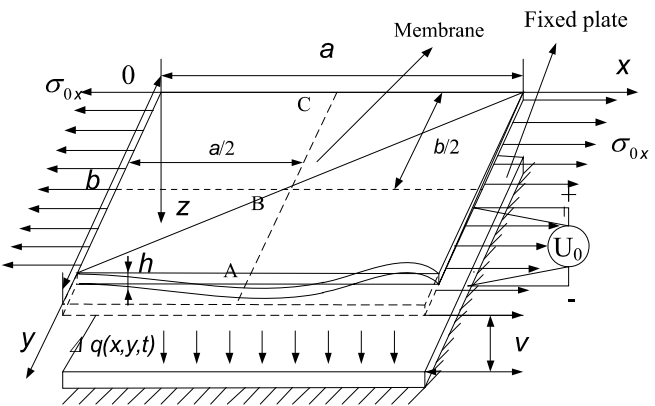

b) Dynamics model of micro film

Fig. 2. Multi-field coupled dynamics model of micro film resonant sensor

$\Delta q$ is the force per unit area on the film, including the electrostatic force and the Casimir force, it can be given as:

$\Delta q=\Delta q_{0}+\Delta q_{r}$

From the Lifshitz formula, the Casimir force per unit area is [24]:

$\Delta q_{r}=\frac{\pi^{2} \hbar c}{240(v-w)^{4}}$

where $\hbar$ is the Plank constant divided by $2 \pi, \hbar=1.055 \times 10^{-34} \mathrm{~J} \cdot \mathrm{S} ; c$ is the light speed, $c=2.998 \times 10^{8} \mathrm{~m} / \mathrm{s}$.

Consider the non-linearity of the Casimir force, the Casimir force is written as a Gaussian expansion at the static mean displacement, and letting $\varepsilon_{1}=\bar{w}_{0} / v$, ones can obtain:

$\Delta q_{r}=\frac{\pi^{2} \hbar c}{60\left(v-\bar{w}_{0}\right)^{5}} \Delta w+\frac{\pi^{2} \hbar c \varepsilon_{1}}{12 v^{5} \bar{w}_{0}} \Delta w^{2}+\frac{\pi^{2} \hbar c \varepsilon_{1}}{4 v^{6} \bar{w}_{0}} \Delta w^{3}+\cdots$

The dynamic electrostatic force per unit area due to the film displacement is [25]:

$\Delta q_{0}=\frac{U_{0}^{2} \varepsilon_{0}}{(v-w)^{3}} \cdot \Delta w$

where $\varepsilon_{0}$ is the permittivity constant of free space, $\varepsilon_{0}=8.85 \times 10^{-12} c^{2} \cdot \mathrm{N}^{-1} \cdot \mathrm{m}^{-2}$.

Considering nonlinearity of electrostatic force, the dynamic electrostatic force on the film is written as a Gaussian expansion at the static mean displacement, one obtains 
$\Delta q_{0}=\frac{U_{0}^{2} \varepsilon_{0}}{\left(v-\bar{w}_{0}\right)^{3}} \Delta w+\frac{3 U_{0}^{2} \varepsilon_{0} \varepsilon_{1}}{v^{3} \bar{w}_{0}} \Delta w^{2}+\frac{6 U_{0}^{2} \varepsilon_{0} \varepsilon_{1}}{v^{4} \bar{w}_{0}} \Delta w^{3}+\cdots$

Thus, the force per unit area on the film is:

$$
\begin{aligned}
\Delta q & =\left[\frac{U_{0}^{2} \varepsilon_{0}}{\left(v-\bar{w}_{0}\right)^{3}}+\frac{\pi^{2} \hbar c}{60\left(v-\bar{w}_{0}\right)^{5}}\right] \Delta w+\left[\frac{3 U_{0}^{2} \varepsilon_{0}}{v^{3} \bar{w}_{0}}+\frac{\pi^{2} \hbar c}{12 v^{5} \bar{w}_{0}}\right] \varepsilon_{1} \Delta w^{2} \\
& +\left[\frac{6 U_{0}^{2} \varepsilon_{0}}{v^{4} \bar{w}_{0}}+\frac{\pi^{2} \hbar c}{4 v^{6} \bar{w}_{0}}\right] \varepsilon_{1} \Delta w^{3} .
\end{aligned}
$$

Substituting Eq. (9) into Eq. (1), one obtains:

$$
\begin{aligned}
& \rho h \frac{\partial^{2} \Delta w}{\partial t^{2}}+\gamma \frac{\partial w}{\partial t}-\left(\sigma_{x 0}+\frac{\partial^{2} \varphi}{\partial y^{2}}\right) h \frac{\partial^{2} \Delta w}{\partial x^{2}}-\left(\sigma_{y 0}+\frac{\partial^{2} \varphi}{\partial x^{2}}\right) h \frac{\partial^{2} \Delta w}{\partial y^{2}} \\
& \quad=\left[\frac{U_{0}^{2} \varepsilon_{0}}{\left(v-\bar{w}_{0}\right)^{3}}+\frac{\pi^{2} \hbar c}{60\left(v-\bar{w}_{0}\right)^{5}}\right] \Delta w+\left[\frac{3 U_{0}^{2} \varepsilon_{0}}{v^{3} \bar{w}_{0}}+\frac{\pi^{2} \hbar c}{12 v^{5} \bar{w}_{0}}\right] \varepsilon_{1} \Delta w^{2} \\
& \quad+\left[\frac{6 U_{0}^{2} \varepsilon_{0}}{v^{4} \bar{w}_{0}}+\frac{\pi^{2} \hbar c}{4 v^{6} \bar{w}_{0}}\right] \varepsilon_{1} \Delta w^{3} .
\end{aligned}
$$

For the boundary conditions, the solutions of Eqs. (1) and (2) can be given as [26]:

$$
\begin{aligned}
& \Delta w(x, y, t)=W(x, y) T(t), \\
& \varphi(x, y, t)=\phi(x, y) T^{2}(t), \\
& W(x, y)=\sin \frac{m \pi x}{a} \cos \frac{n \pi y}{b} .
\end{aligned}
$$

Thus:

$$
\Delta w(x, y)=\sin \frac{m \pi x}{a} \cos \frac{n \pi y}{b} T(t)
$$

Substituting Eqs. (12) and (14) into Eq. (2), one yields:

$$
\frac{1}{E_{1}} \frac{\partial^{4} \phi}{\partial y^{4}}+\frac{1}{E_{2}} \frac{\partial^{4} \phi}{\partial x^{4}}=\frac{m^{2} n^{2} \pi^{4}}{2 a^{2} b^{2}}\left(\cos \frac{2 m \pi x}{a}-\cos \frac{2 n \pi y}{b}\right) .
$$

Let:

$$
\phi(x, y)=\alpha \cos \frac{2 m \pi x}{a}-\beta \cos \frac{2 n \pi y}{b} .
$$

Substituting it into Eq. (15), one yields the following equations:

$$
\alpha=\frac{E_{2} n^{2} a^{2}}{32 m^{2} b^{2}}, \quad \beta=\frac{E_{1} m^{2} b^{2}}{32 n^{2} a^{2}} .
$$

Substituting Eq. (17) into Eq. (12), one yields:

$$
\varphi(x, y, t)=\left(\frac{E_{2} n^{2} a^{2}}{32 m^{2} b^{2}} \cos \frac{2 m \pi x}{a}-\frac{E_{1} m^{2} b^{2}}{32 n^{2} a^{2}} \cos \frac{2 n \pi y}{b}\right) T^{2}(t) .
$$


The substitution of Eqs. (14) and (18) into Eq. (1) and using the Galerkin method [27], one obtains:

$$
\begin{aligned}
\iint & {\left[\rho h \frac{\partial^{2} \Delta w}{\partial t^{2}}+\gamma \frac{\partial \Delta w}{\partial t}-\left(\sigma_{0 x}+\frac{\partial^{2} \varphi}{\partial y^{2}}\right) h \frac{\partial^{2} w}{\partial x^{2}}-\left(\sigma_{0 y}+\frac{\partial^{2} \varphi}{\partial x^{2}}\right) h \frac{\partial^{2} \Delta w}{\partial y^{2}}\right] } \\
& \cdot \sin \frac{m \pi x}{a} \cos \frac{n \pi y}{b} d x d y \\
& =\iint\left\{\left[\frac{U_{0}{ }^{2} \varepsilon_{0}}{\left(v-\bar{w}_{0}\right)^{3}}+\frac{\pi^{2} \hbar c}{60\left(v-\bar{w}_{0}\right)^{5}}\right] \Delta w+\left[\frac{3 U_{0}{ }^{2} \varepsilon_{0}}{v^{3} \bar{w}_{0}}+\frac{\pi^{2} \hbar c}{12 v^{5} \bar{w}_{0}}\right] \varepsilon_{1} \Delta w^{2}\right. \\
& \left.+\left[\frac{6 U_{0}^{2} \varepsilon_{0}}{v^{4} \bar{w}_{0}}+\frac{\pi^{2} \hbar c}{4 v^{6} \bar{w}_{0}}\right] \varepsilon_{1} \Delta w^{3}\right\} \sin \frac{m \pi x}{a} \cos \frac{n \pi y}{b} d x d y .
\end{aligned}
$$

Let it be that:

$$
\begin{aligned}
S_{111} & =\iint \rho h \sin ^{2} \frac{m \pi x}{a} \cos ^{2} \frac{n \pi y}{b} d x d y=\frac{\rho h a b}{4}, \\
S_{222} & =\iint \gamma \sin ^{2} \frac{m \pi x}{a} \cos ^{2} \frac{n \pi y}{b} d x d y=\frac{\gamma a b}{4}, \\
S_{333} & =\iint\left(\sigma_{0 x} \frac{\partial^{2} W}{\partial x^{2}}+\sigma_{0 y} \frac{\partial^{2} W}{\partial y^{2}}\right) h \sin \frac{m \pi x}{a} \cos \frac{n \pi y}{b} d x d y \\
& =-\frac{\pi^{2} a b h}{4}\left(\frac{m^{2}}{a^{2}} \sigma_{0 x}+\frac{n^{2}}{b^{2}} \sigma_{0 y}\right), \\
S_{444} & =\iint\left(\frac{\partial^{2} \phi}{\partial y^{2}} \frac{\partial^{2} W}{\partial x^{2}}+\frac{\partial^{2} \phi}{\partial x^{2}} \frac{\partial^{2} W}{\partial y^{2}}\right) h \sin \frac{m \pi x}{a} \cos \frac{n \pi y}{b} d x d y \\
= & \frac{3 \pi^{4} a b h}{64}\left(\frac{E_{1} m^{4}}{a^{4}}-\frac{E_{2} n^{4}}{b^{4}}\right), \\
S_{555} & =\iint\left[\frac{U_{0}{ }^{2} \varepsilon_{0}}{\left(v-\bar{w}_{0}\right)^{3}}+\frac{\pi^{2} \hbar c}{60\left(v-\bar{w}_{0}\right)^{5}}\right] \sin { }^{2} \frac{m \pi x}{a} \cos ^{2} \frac{n \pi y}{b} d x d y \\
= & \frac{U_{0}{ }^{2} \varepsilon_{0} a b}{4\left(v-\bar{w}_{0}\right)^{3}}+\frac{\pi^{2} \hbar c a b}{240\left(v-\bar{w}_{0}\right)^{5}}, \\
S_{666} & =\iint\left(\frac{6 U_{0}{ }^{2} \varepsilon_{0}}{v^{4} \bar{w}_{0}}+\frac{\pi^{2} \hbar c}{4 v^{6} \bar{w}_{0}}\right) \varepsilon_{1} \sin ^{3} \frac{m \pi x}{a} \cos ^{3} \frac{n \pi y}{b} d x d y=0, \\
S_{777} & =\iint\left(\frac{6 U_{0}{ }^{2} \varepsilon_{0}}{v^{4} \bar{w}_{0}}+\frac{\pi^{2} \hbar c}{4 v^{6} \bar{w}_{0}}\right) \varepsilon_{1} \sin ^{4} \frac{m \pi x}{a} \cos ^{4} \frac{n \pi y}{b} d x d y=\frac{9 a b \varepsilon_{1}}{64}\left(\frac{6 U_{0}{ }^{2} \varepsilon_{0}}{v^{4} \bar{w}_{0}}+\frac{\pi^{2} \hbar c}{4 v^{6} \bar{w}_{0}}\right) .
\end{aligned}
$$

Thus, Eq. (19) can be changed into the following form:

$$
S_{111} T^{\prime \prime}+S_{222} T^{\prime}-S_{333} T-S_{444} T^{3}=S_{555} T+S_{777} T^{3} .
$$

Utilizing the KBM perturbation method [28], let, $\varepsilon_{2}=h^{2} / a b$ and then:

$T^{\prime \prime}+\frac{\left(-S_{333}-S_{555}\right)}{S_{111}} T=\varepsilon_{2}\left(\frac{S_{777} a b}{S_{111} h^{2}} T^{3}+\frac{-S_{222} a b}{S_{111} h^{2}} T^{\prime}\right)$.

In order to facilitate the calculation, Eq. (22) is converted into following form:

$T^{\prime \prime}+\omega_{0}^{2} T=\varepsilon_{2}\left(\zeta_{1} T^{3}+\zeta_{2} T^{\prime}\right)$. 
Here:

$\omega_{0}^{2}=\frac{\left(-S_{333}-S_{555}\right)}{S_{111}}$,

$\zeta_{1}=\frac{S_{777} a b}{S_{111} h^{2}}$

$\zeta_{2}=\frac{-S_{222} a b}{S_{111} h^{2}}$.

Let it be that:

$f\left(T, T^{\prime}\right)=\left(\zeta_{1} T^{3}+\zeta_{2} T^{\prime}\right), \vartheta=\omega_{0} t+\eta$.

Here $\eta$ is the initial phase of film vibration (let $\eta=0$ ).

If $\varepsilon_{2}=0$, Eq. (23) can be changed into following form:

$\frac{d T}{d t}=-k \omega_{0} \sin \vartheta$

Consider the influence of non-linearity, ones assume:

$T=k \cos \vartheta+\varepsilon T_{1}(k, \vartheta)+\varepsilon^{2} T_{2}(k, \vartheta)+\cdots$

Here $k$ and $\vartheta$ is the time function, $T_{1}$ and $T_{2}$ is the power series function of $\varepsilon$.

The equivalent damping ratio $\delta_{e}$ and the equivalent natural frequency $\omega$ of the nonlinear system can be expressed as the form of a small parameter power series:

$\delta_{e}=\varepsilon \delta_{1}(k)+\varepsilon^{2} \delta_{2}(k)+\cdots$,

$\omega=\omega_{0}+\varepsilon \omega_{1}(k)+\varepsilon^{2} \omega_{2}(k)+\cdots$.

Utilizing KBM perturbation method (see Appendix), ones obtain:

$$
\begin{aligned}
& \delta_{1}=\frac{\zeta_{2}}{2}, \quad \omega_{1}=-\frac{3 \zeta_{1} k^{2}}{8 \omega_{0}}, \\
& T_{1}=-\frac{\zeta_{1} k^{3}}{32 \omega_{0}^{2}} \cos 3 \vartheta \\
& \delta_{2}=-\frac{3 \zeta_{1} \zeta_{2} k^{3}}{16 \omega_{0}^{2}}, \omega_{2}=\frac{3 \zeta_{1}^{2} k^{4}}{256 \omega_{0}^{3}}-\frac{\zeta_{2}^{2}}{4 \omega_{0}}-\frac{9 \zeta_{1}^{2} k^{4}}{128 \omega_{0}{ }^{3}}, \\
& T_{2}=\frac{3 \zeta_{1}^{2} k^{5}}{512 \omega_{0}^{4}} \cos 3 \vartheta+\frac{3 \zeta_{1}^{2} k^{5}}{3072 \omega_{0}^{4}} \cos 5 \vartheta .
\end{aligned}
$$

From Eq. (29), the non-linear vibration frequency can be obtained:

$\omega=\omega_{0}-\frac{3 \varepsilon \zeta_{1} k_{0}^{2} e^{\varepsilon \zeta_{2} t}}{8 \omega_{0}}-\frac{15 \varepsilon^{2} \zeta_{1}^{2} k_{0}{ }^{4} e^{2 \varepsilon \zeta_{2} t}}{256 \omega_{0}{ }^{3}}-\frac{\varepsilon^{2} \zeta_{2}^{2}}{4 \omega_{0}}$.

Substituting Eq. (31) and Eq. (33) into Eq. (27), one yields:

$$
\begin{aligned}
T & =k \cos \vartheta+\varepsilon T_{1}+\varepsilon^{2} T_{2} \\
& =k \cos \vartheta+\left(\frac{3 \varepsilon^{2} \zeta_{1}^{2} k^{5}}{512 \omega_{0}^{4}}-\frac{\varepsilon \zeta_{1} k^{3}}{32 \omega_{0}^{2}}\right) \cos 3 \vartheta+\frac{3 \varepsilon^{2} \zeta_{1}^{2} k^{5}}{3072 \omega_{0}{ }^{4}} \cos 5 \vartheta
\end{aligned}
$$


From Eq. (14) and Eq. (35), the displacement function of nonlinear vibration of rectangular orthotropic film with damping is obtained:

$$
\begin{aligned}
& w(x, y, t)=\sum_{m=1}^{\infty} \sum_{n=1}^{\infty} \sin \frac{m \pi x}{a} \cos \frac{n \pi y}{b} k \cos \vartheta \\
& +\left(\frac{3 \varepsilon^{2} \zeta_{1}^{2} k^{5}}{512 \omega_{0}^{4}}-\frac{\varepsilon \zeta_{1} k^{3}}{32 \omega_{0}^{2}}\right) \cos 3 \vartheta+\frac{3 \varepsilon^{2} \zeta_{1}^{2} k^{5}}{3072 \omega_{0}^{4}} \cos 5 \vartheta
\end{aligned}
$$

\section{Results and discussions}

The parameters of the micro film are shown in Table 1 (here, $k_{0}=2 \times 10^{-7} \mathrm{~m}, U_{0}=0.1 v$, $\sigma_{0 y}=0$ ), Table 2 gives the changes of nonlinear frequencies (here, $\varepsilon=0.001$ ), $\omega_{0}$ is the nonlinear frequencies of the micro film without considering the Casimir force, $\omega_{1}$ is the nonlinear frequencies of the micro film with considering the Casimir force, $\eta$ is the relative deviations between them. Fig. 2 gives the comparisons of the first four orders of the nonlinear frequencies for different nonlinear parameters. Fig. 3 gives nonlinear frequency changes with the main parameters.

Table 1. System parameters

\begin{tabular}{|c|c|c|c|c|c|c|c|}
\hline$a(\mathrm{~mm})$ & $b(\mathrm{~mm})$ & $h(\mu \mathrm{m})$ & $v(\mu \mathrm{m})$ & $\varepsilon_{0}\left(c^{2} \cdot \mathrm{N}^{-1} \cdot \mathrm{m}^{-2}\right)$ & $E_{1}(\mathrm{GPa})$ & $E_{2}(\mathrm{GPa})$ & $\rho\left(\mathrm{kg} / \mathrm{m}^{3}\right)$ \\
\hline 0.8 & 0.3 & 1 & 0.3 & $8.85 \times 10^{-12}$ & 1.4 & 0.9 & 2330 \\
\hline
\end{tabular}

From Table 2, Fig. 3 and Fig. 4, the following observations are worth noting:

1) Nonlinear resonant frequencies without considering the Casimir force are larger than those with considering the Casimir force. The Casimir force and electrostatic force correspond to two springs in series, so the Casimir force can lead to a decrease of the nonlinear resonant frequencies just like the electrostatic force.

2) For $\varepsilon=0.001$, the deviation between the nonlinear frequencies of the micro sensor with and without the Casimir force decreases with increasing the order number of the mode. For the first order frequency, the deviation between the nonlinear frequencies is $19.51 \%$, for the fourth order one, the deviation between the nonlinear frequencies is $0.796 \%$. It shows that the Casimir force has a greater influence on the low-order nonlinear frequencies. So, the Casimir force should be considered when determining the low order of the resonant frequencies for the micro film.

3) As the nonlinear parameter increases, the first four order nonlinear frequencies decrease, and the deviation between the nonlinear frequencies with and without considering the Casimir force decreases. The deviation between the nonlinear frequencies with considering the Casimir force is larger than that without considering the Casimir force. It shows that nonlinearity of the Casimir force has an effect on the resonant frequency of the micro film, but the nonlinearity of the electrostatic force has much larger effect on the resonant frequency of the micro film when the nonlinearity is stronger.

4) As the film length increases, the deviation between the nonlinear frequencies for $\varepsilon=0$ and $\varepsilon=0.01$ increases significantly. The deviation between the nonlinear frequencies of the micro sensor with considering the Casimir force is larger than those without considering the Casimir force. It is because the effects of the Casimir force become large when the film length is large.

5) As the initial clearance between the micro film and base plate increases, the deviation between the nonlinear frequencies with and without considering the Casimir force decreases. When the initial clearance is above $400 \mathrm{~nm}$, the nonlinear frequencies with and without considering the Casimir force are nearly identical to each other. It shows that the Casimir force should be considered when the initial clearance is below $400 \mathrm{~nm}$. It is because the Casimir force is inversely proportional to the fourth power of the initial clearance. 
Table 2. Fluctuations of nonlinear frequencies

\begin{tabular}{|c|c|c|c|c|}
\hline & Mode $(1,1)$ & Mode $(2,2)$ & Mode $(3,3)$ & Mode $(4,4)$ \\
\hline$\omega_{0}(\mathrm{rad} / \mathrm{s})$ & 157596.58 & 338485.24 & 513937.67 & 688124.72 \\
\hline$\omega_{1}(\mathrm{rad} / \mathrm{s})$ & 131863.64 & 327295.31 & 506637.69 & 682689.83 \\
\hline$\eta(\%)$ & 19.51 & 3.419 & 1.441 & 0.7961 \\
\hline
\end{tabular}

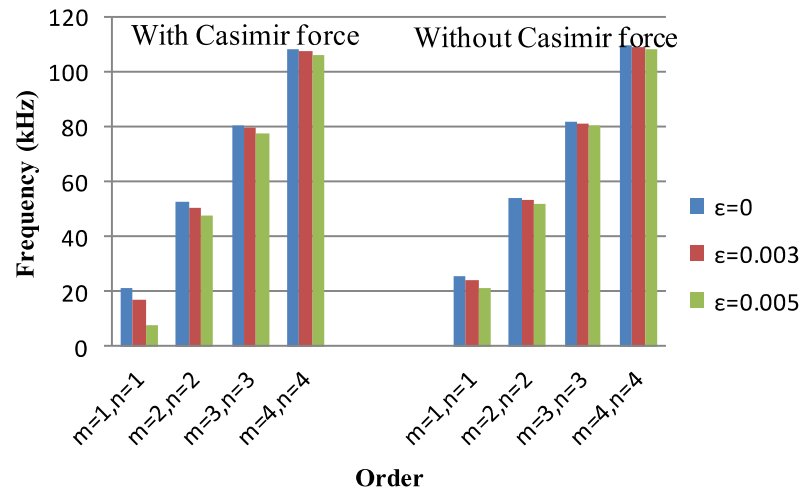

Fig. 3. Frequencies for different nonlinear parameters $\varepsilon$

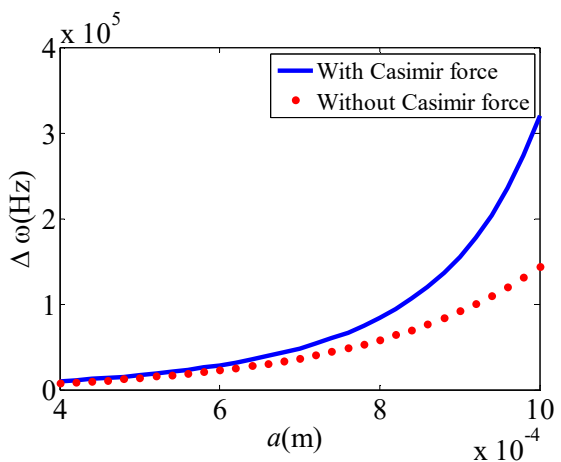

a) $a$ changes

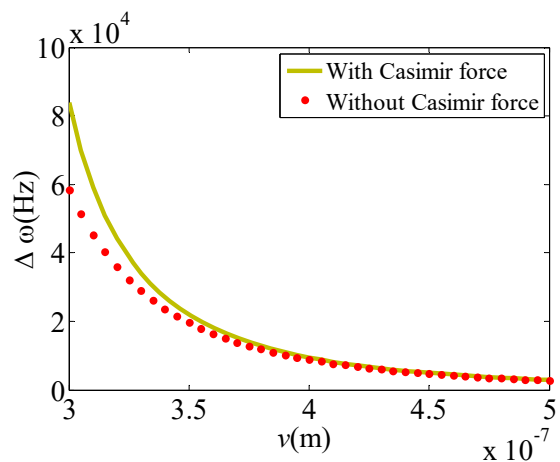

b) $v$ changes

Fig. 4. Nonlinear frequency error changes with parameters

The effects of the Casimir force, small parameter and air damping force on the nonlinear vibrating amplitudes are investigated for the mode $(1,1)$ (see Fig. 5, Fig. 6 and Fig. 7). Fig. 5 gives the vibrating amplitudes of nonlinear free vibration at $\varepsilon=0.01$ with considering the Casimir force. Fig. 6 gives the difference of the vibrating amplitudes with and without considering the Casimir force $(\varepsilon=0.01)$. Fig. 7 gives the vibrating amplitudes of nonlinear free vibration for $\varepsilon=0.01$ and $\varepsilon=0$ (considering the Casimir force).

From Fig. 5, Fig. 6 and Fig. 7, the following observations are worth noting:

1) The displacement time trace of free vibration at each point on the micro film reduces periodically with the time. Due to the existence of air damping, the amplitude eventually approaches zero.

2) The vibration amplitude of the center point $B$ is much smaller than that of point $A$ and point C. This is because the distances between the center point B and two free film edges are equal, and this makes part of the vibrations cancel each other after superimposing on the center point $\mathrm{B}$. For point $\mathrm{A}$ and point $\mathrm{C}$, the nonlinear vibration direction is opposite. This is due to the fact that two points are symmetrical about the film midpoint, and the motions of two points mainly depend on the first order of the vibrating mode. 


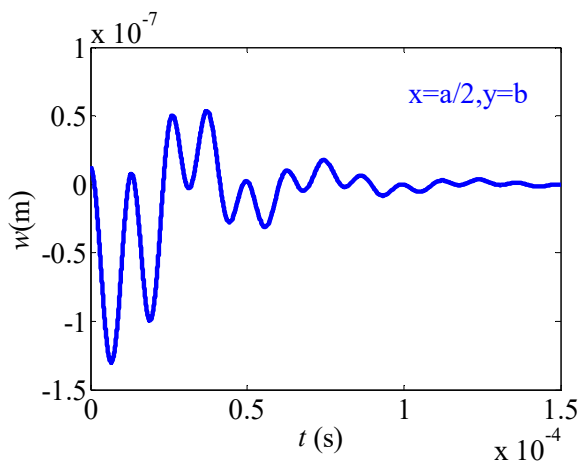

a) Point $\mathrm{A}(x=a / 2, y=b)$

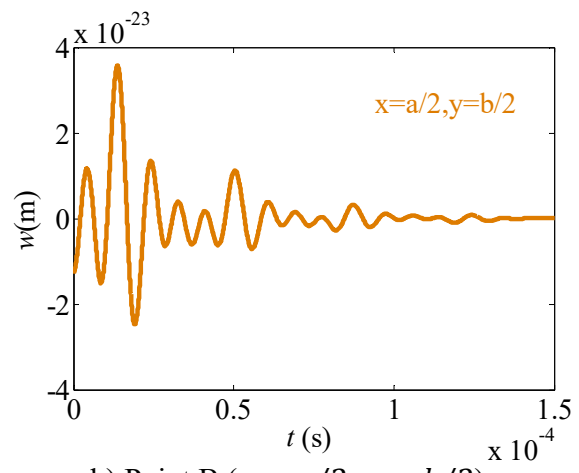

b) Point $\mathrm{B}(x=a / 2, y=b / 2)$

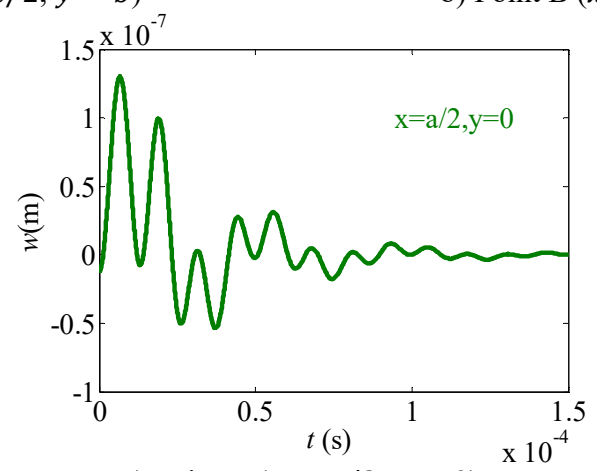

c) Point $\mathrm{C}(x=a / 2, y=0)$

Fig. 5. Displacement variation of nonlinear free vibration

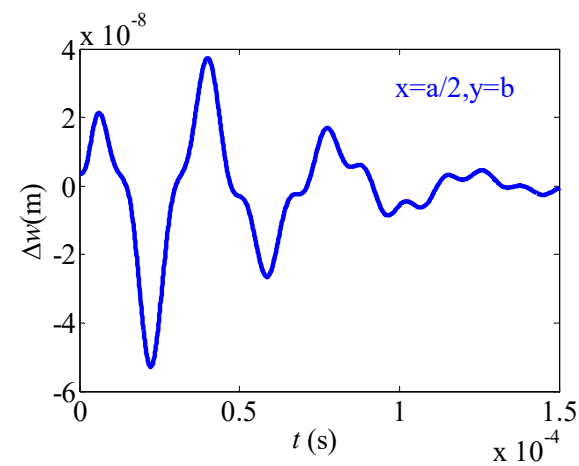

a) Point $\mathrm{A}(x=a / 2, y=b)$

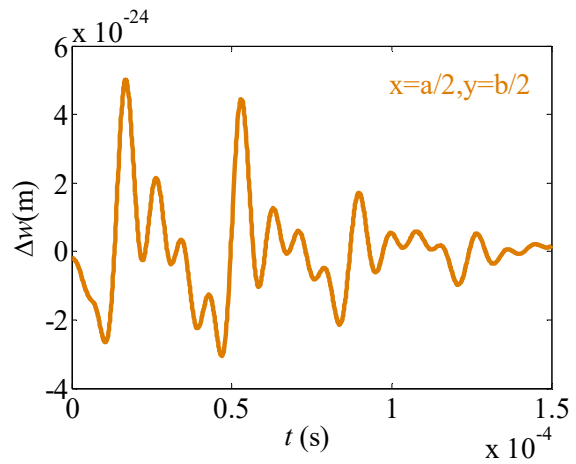

b) Point B $(x=a / 2, y=b / 2)$

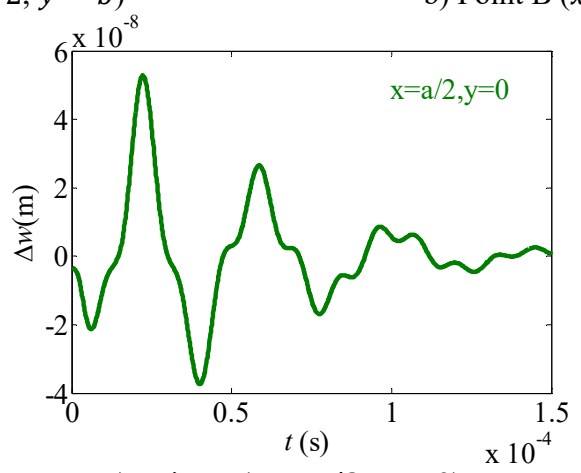

c) Point $\mathrm{C}(x=a / 2, y=0)$

Fig. 6. Effects of Casimir force on difference of nonlinear free vibration $(\varepsilon=0.01)$ 


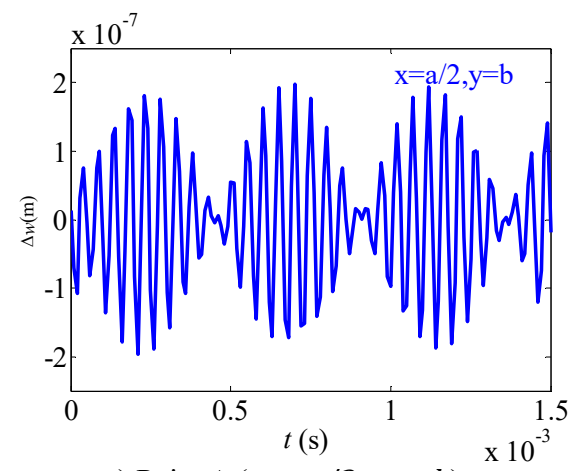

a) Point $\mathrm{A}(x=a / 2, y=b)$

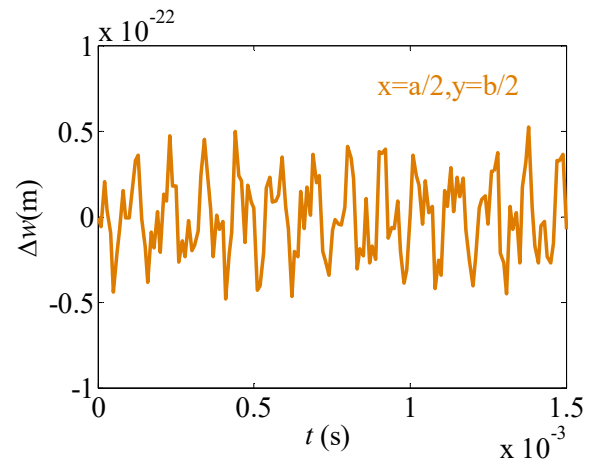

b) Point $\mathrm{B}(x=a / 2, y=b / 2)$

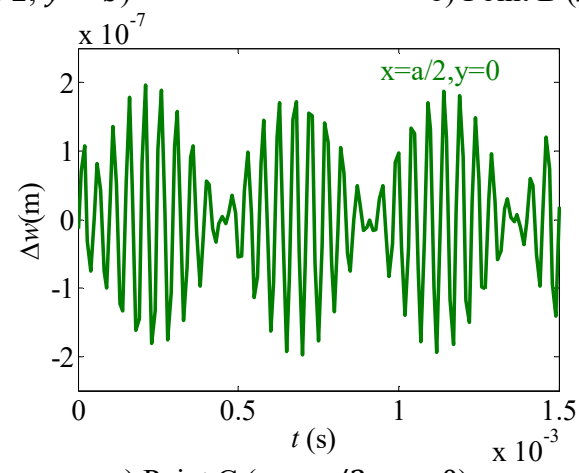

c) Point $\mathrm{C}(x=a / 2, y=0)$

Fig. 7. Effects of small parameter on difference of nonlinear free vibration

3) The difference between the vibrating amplitudes with and without considering the Casimir force reduces periodically as well. It shows that the Casimir force have an important effect on vibration amplitude of the micro film. For the point $\mathrm{A}$ and point $\mathrm{C}$, the difference between the vibrating amplitudes with and without considering the Casimir force gets to $50 \%$ of its vibration amplitude. For the point $\mathrm{B}$, the difference between the vibrating amplitudes with and without considering the Casimir force is above $10 \%$ of its vibration amplitude. It shows that the Casimir force should be considered when determining the vibration amplitudes of the micro film.

4) The difference between the vibrating amplitudes for $\varepsilon=0.01$ and $\varepsilon=0$ changes periodically for the points $\mathrm{A}, \mathrm{B}$ point $\mathrm{C}$. The difference does not reduce with the time. It shows that a high order of the harmonic vibrations on the micro film does not decay with the time.

To illustrate the above mentioned analysis about the nonlinear frequencies of the micro film, a micro film resonant sensor is fabricated by lithography, etching and other processes (see Fig. 8(a)). The detection of the natural frequencies for the micro resonant sensor is done. Here, the detecting method of electrostatic excitation and capacitance measurement is used to measure the vibration frequency. When the input signal frequency is close to the resonant frequency of the film, the film resonance occurs. Fig. 8 shows the sensor and its measuring system. Fig. 9 gives detection results of the micro film for two different size sensors (see Table 3). Table 4 gives the comparison between measured results and calculative values ( $\eta$ is the relative deviation of measured results and calculative values).

Table 3. Parameters of measured micro-film

\begin{tabular}{|c|c|c|c|c|c|c|c|c|c|}
\hline \multicolumn{4}{|c|}{ Small size sensor } & \multicolumn{5}{c|}{ Large size sensor } \\
\hline$a_{1}(\mathrm{~mm})$ & $b_{1}(\mathrm{~mm})$ & $v_{1}(\mu \mathrm{m})$ & $h_{1}(\mu \mathrm{m})$ & $U_{0}(v)$ & $a_{2}(\mathrm{~mm})$ & $b_{2}(\mathrm{~mm})$ & $v_{2}(\mu \mathrm{m})$ & $h_{2}(\mu \mathrm{m})$ & $U_{0}(v)$ \\
\hline 0.96 & 0.31 & 0.3 & 10 & 0.1 & 1.50 & 0.61 & 1 & 10 & 0.1 \\
\hline
\end{tabular}


Table 4. Comparison between measured results and calculative values

\begin{tabular}{|c|c|c|c|c|}
\hline & Test results & $\begin{array}{c}\text { With Casimir force } \\
(\varepsilon=0.001)\end{array}$ & $\begin{array}{c}\text { With Casimir force } \\
(\varepsilon=0)\end{array}$ & $\begin{array}{c}\text { Without Casimir force } \\
(\varepsilon=0)\end{array}$ \\
\hline \multicolumn{5}{|c|}{ Small size sensor } \\
\hline \begin{tabular}{c} 
Frequency (kHz) \\
\cline { 2 - 5 } $\begin{array}{c}\text { Relative deviation } \\
\eta(\%)\end{array}$
\end{tabular} & 13.8421 & 14.3178 & 15.1149 & 20.1341 \\
\hline \multicolumn{5}{|c|}{ Large size sensor } \\
\hline Frequency (kHz) & 13.9737 & 14.6214 & 9.195 & 45.46 \\
\hline $\begin{array}{c}\text { Relative deviation } \\
\eta(\%)\end{array}$ & 4.635 & 4.636 & 14.6258 & 4.6353 \\
\hline
\end{tabular}

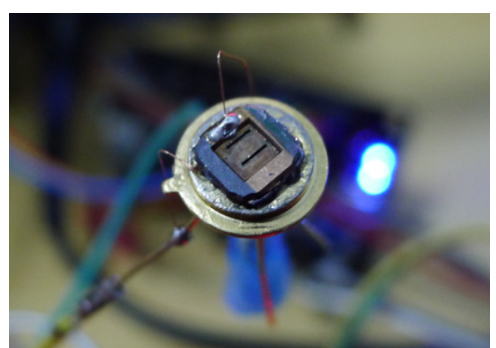

a) Resonator

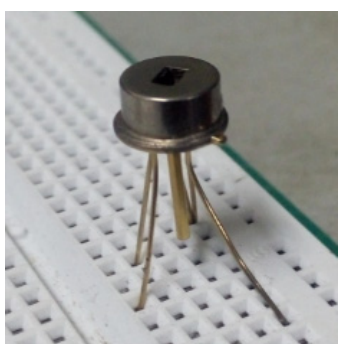

b) Sensor

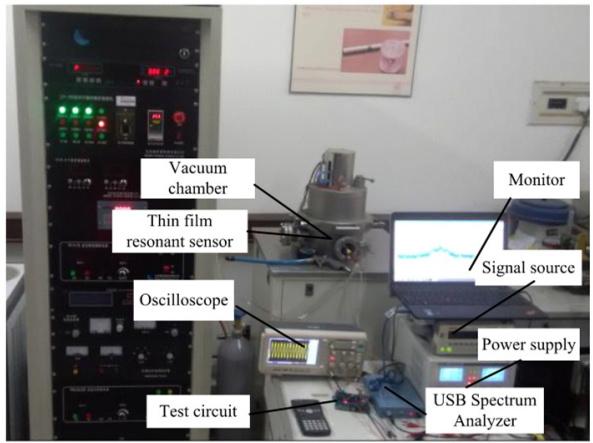

c) Measuring system

Fig. 8. Micro film resonant sensor and its measuring system

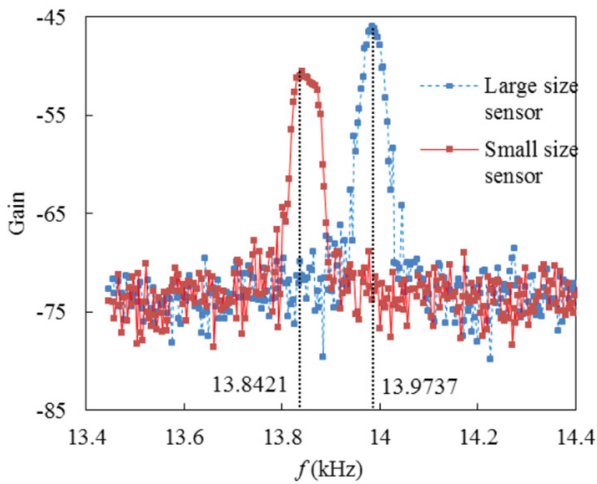

Fig. 9. Frequency test of micro film for two different size sensors

Results show:

1) For a small size sensor, the relative deviation between the measured results and calculative 
values is $3.436 \%$ (with the Casimir force and $\varepsilon=0.001$ ). For a large size sensor, the relative deviation between the measured results and calculative values is $4.635 \%$ (with the Casimir force and $\varepsilon=0.001)$. The measuring frequencies of the micro film are in good agreement with the calculative values.

2) For a small size sensor, the relative deviations between the measured results and calculative values are $3.436 \%$ (with the Casimir force and $\varepsilon=0.001$ ), $9.195 \%$ (with the Casimir force and $\varepsilon=0$ ) and $45.46 \%$ (without the Casimir force and $\varepsilon=0$ ). For a large size sensor, the relative deviations between the measured results and calculative values are $4.635 \%$ (with the Casimir force and $\varepsilon=0.001$ ), $4.667 \%$ (with the Casimir force and $\varepsilon=0$ ) and $4.734 \%$ (without the Casimir force and $\varepsilon=0$ ). Considering the Casimir force and nonlinear parameter, the calculated natural frequency is more near to the test results.

The authors declare that there is no conflict of interests regarding the publication of this article.

\section{Conclusions}

In this paper, a multi-field coupled dynamics model of the micro film resonator is proposed. Here, the Casimir force, air damping force, elastic force and electrostatic force are considered. Using a model, the nonlinear vibration frequency and displacement response of the micro film resonator are investigated. Results show:

1) The Casimir force has an important effect on the vibration frequencies of the micro resonant film. When the clearance between the film and baseplate decreases, the effect becomes more significant. When the clearance is below $400 \mathrm{~nm}$, the effect should be considered.

2) When the size of the film increases, the effect of the Casimir force on the nonlinear frequencies of the micro resonant film becomes more significant as well.

3) The Casimir force has a significant important effect on the vibrating amplitudes of the micro film. When determining the vibrating amplitudes of the micro film, the Casimir force should not be neglected.

4) Effects of the Casimir force can be illustrated by detecting the resonant frequency difference of the micro film under different initial clearances.

\section{Acknowledgements}

This Project is supported by the Key Basic Research Foundation in the Hebei Province of China (13961701D) and Graduate Innovation Fund of Hebei Province (CXZZBS2017043).

\section{References}

[1] Thielicke E., Obermeier E. Microactuators and their technologies. Mechatronics, Vol. 10, Issues 4-5, 2000, p. 431-455.

[2] Rafael N. G., Anna M. B., Alfons D. AC transfer function of electrostatic capacitive sensors based on 1-D equivalent model: application to silicon microphones. Journal of Microelectromechanical Systems, Vol. 12, Issue 6, 2003, p. 972-978.

[3] Yuan W., Ren S., Deng J., et al. Review of silicon micromachined resonant pressure sensor. Journal of Mechanical Engineering, Vol. 49, Issue 20, 2013, p. 2-9.

[4] Kumar D. D., Kumar N., Kalaiselvam S., et al. Micro-tribo-mechanical properties of nanocrystalline TiN thin films for small scale device applications. Tribology International, Vol. 88, 2015, p. 25-30.

[5] Gerhard M., Sebastian B., Sumit P. Novel chemical sensor applications in commercial aircraft. Procedia Engineering, Vol. 25, 2011, p. 16-22.

[6] Lizhong Xu, Xiaorui Fu Electromechanical fluidic coupled vibrations for micro film considering van der Waals force. Journal of Vibroengineering, Vol. 17, Issue 1, 2015, p. 455-467.

[7] Lin W. Free vibration analysis of plane membranes by finite element method. Journal of Dynamics and Control, 2010. 
[8] García-Alonso A., García J., Castaño E., et al. Strain sensitivity and temperature influence on sputtered thin films for piezo-resistive sensors. Sensors and Actuators A: Physical, Vols. 37-38, Issue 38, 1993, p. 784-789.

[9] Fan S. Investigation on nonlinear vibration characteristics of resonant silicon microstructure pressure sensor. Chinese Journal of Scientific Instrument, Vol. 27, Issue 12, 2006, p. 1670-1673.

[10] Capasso F., Munday J. N., Iannuzzi D., et al. Casimir forces and quantum electrodynamical torques: physics and nanomechanics. IEEE Journal of Selected Topics in Quantum Electronics, Vol. 13, Issue 2, 2007, p. 400-414.

[11] Reutskiy S. Y. Vibration analysis of arbitrarily shaped membranes. Computer Modeling in Engineering and Sciences, Vol. 51, Issue 2, 2009, p. 115-142.

[12] Gonçalves P. B., Soares R. M., Pamplona D. Nonlinear vibrations of radially stretched circular hyperelastic membrane. Journal of Sound and Vibration, Vol. 327, Issues 1-2, 2009, p. 231-248.

[13] Changjiang Liu, Zhoulian Zheng, Long Jun, et al. Dynamic analysis for nonlinear vibration of pre-stressed orthotropic membranes with viscous damping. International Journal of Structural Stability and Dynamics, Vol. 13, Issue 2, 2013, p. 60-66.

[14] Sorokin V. M., Yashchenko A. K. Vibrations of frameless film membrane stabilized by Ampère force in zero gravity. Journal of Applied Mechanics and Technical Physics, Vol. 54, Issue 54, 2013, p. 885-893.

[15] Weber J., Link M., Primig R., et al. Sensor for ambient pressure and material strains using thin film bulk acoustic resonator. Ultrasonics Symposium, 2005, p. 1258-1261.

[16] Uhlig S., Rau S., Schultes G. Piezoresistivity of polycrystalline silicon applying AIC process-route. Sensors and Actuators A: Physical, Vol. 172, Issue 2, 2011, p. 447-454.

[17] Zhao X., Ashley G. M., Garcia-Gancedo L., et al. Protein functionalized ZnO thin film bulk acoustic resonator as odorant biosensor. Sensors and Actuators B Chemical, Vol. 163, Issue 1, 2012, p. 242.

[18] Jiang Y., Kanda K., Iga Y., et al. Monolithic integration of $\mathrm{Pb}(\mathrm{Zr}, \mathrm{Ti}) \mathrm{O}_{3}$, thin film based resonators using complete dry microfabrication process. Microsystem Technologies, Vol. 19, Issue 1, 2013, p. $137-142$.

[19] Gualdino A., Chu V., Conde J. P. Study of out-of-plane vibrational modes in thin-film amorphous silicon micromechanical disk resonators. Journal of Applied Physics, Vol. 113, Issue 17, 2013, p. 213506.

[20] Ren S., Yuan W., Qiao D., et al. Micromachined pressure sensor with integrated resonator operating at atmospheric pressure. Sensors, Vol. 13, Issue 12, 2013, p. 17006-17024.

[21] Hamelin B., Daruwalla A., Ayazi F. Stiffness trimming of high Q MEMS resonators by excimer laser annealing of germanium thin film on silicon. International Conference on MICRO Electro Mechanical Systems, 2016, p. 1026-1029.

[22] Alahnomi R. A., Zakaria Z., Ruslan E., et al. High sensitive microwave sensor based on symmetrical split ring resonator for material characterization. Microwave and Optical Technology Letters, Vol. 58, Issue 9, 2016, p. 2106-2110.

[23] Pan J., Ming G. U. Geometric nonlinear effect to square tensioned membrane's free vibration. Journal of Tongji University, 2007.

[24] Geyer B., Klimchitskaya G. L., Mostepanenko V. M. Analytic approach to thermal Casimir force between metaland dielectric. Annals of Physics, Vol. 323, 2008, p. 291-316.

[25] Liu C. J., Zheng Z. L., He X. T., et al. L-P perturbation solution of nonlinear free vibration of pre-stressed orthotropic membrane with large amplitude. Mathematical Problems in Engineering, Vol. 2010, 2010, p. 242-256.

[26] Awrejcewicz J., Krys'ko V. A., Shitikova M. V. Nonclassical thermoelastic problems in nonlinear dynamics of shells: applications of Bubnov-Galerkin and finite difference numerical methods. Applied Mechanics Reviews, Vol. 57, Issue 1, 2012, p. B6-B7.

[27] Shin C., Kim W., Chung J. Free in-plane vibration of axially moving membrane. Journal of Sound and Vibration, Vol. 272, Issue 1, 2004, p. 137-154.

[28] Liu Yanzhu, Chen Wenliang, Chen Liquan Mechanic of Vibrations. Higher Education Press, Beijing, 1998, p. 201-206. 


\section{Appendix}

\section{A1. Solutions of nonlinear equations by KBM method}

In Eq. (26) and Eq. (27), $k$ and $\vartheta$ satisfy the following equations:

$$
\begin{aligned}
& \frac{d k}{d t}=\left[\varepsilon \delta_{1}(k)+\varepsilon^{2} \delta_{2}(k)+\cdots\right] k, \\
& \frac{d \vartheta}{d t}=\omega_{0}+\varepsilon \omega_{1}(k)+\varepsilon^{2} \omega_{2}(k)+\cdots .
\end{aligned}
$$

From Eq. (27), one obtains:

$$
\begin{aligned}
& \frac{d T}{d t}=\frac{d k}{d t}\left(\cos \vartheta+\varepsilon \frac{\partial T_{1}}{\partial k}+\varepsilon^{2} \frac{\partial T_{2}}{\partial k}+\cdots\right)+\frac{d \vartheta}{d t}\left(-k \sin \vartheta+\varepsilon \frac{\partial T_{1}}{\partial \vartheta}+\varepsilon^{2} \frac{\partial T_{2}}{\partial \vartheta}+\cdots\right) \\
& \frac{d^{2} T}{d t^{2}}=\frac{d^{2} k}{d t^{2}}\left(\cos \vartheta+\varepsilon \frac{\partial T_{1}}{\partial k}+\varepsilon^{2} \frac{\partial T_{2}}{\partial k}+\cdots\right)+\frac{d^{2} \vartheta}{d t^{2}}\left(-k \sin \vartheta+\varepsilon \frac{\partial T_{1}}{\partial \vartheta}+\varepsilon^{2} \frac{\partial T_{2}}{\partial \vartheta}+\cdots\right) \\
& +\left(\frac{d k}{d t}\right)^{2}\left(\varepsilon \frac{\partial^{2} T_{1}}{\partial k^{2}}+\varepsilon^{2} \frac{\partial^{2} T_{2}}{\partial k^{2}}+\cdots\right)+2 \frac{d k}{d t} \frac{d \vartheta}{d t}\left(-\sin \vartheta+\varepsilon \frac{\partial^{2} T_{1}}{\partial k \partial \vartheta}+\varepsilon^{2} \frac{\partial^{2} T_{2}}{\partial k \partial \vartheta}+\cdots\right) \\
& +\left(\frac{d \vartheta}{d t}\right)^{2}\left(-k \cos \vartheta+\varepsilon \frac{\partial^{2} T_{1}}{\partial \vartheta^{2}}+\varepsilon^{2} \frac{\partial^{2} T_{2}}{\partial \vartheta^{2}}+\cdots\right) .
\end{aligned}
$$

Similarly, from Eq. (A1) and Eq. (A2), we obtain:

$$
\begin{aligned}
& \frac{d^{2} k}{d t^{2}}=\left[\varepsilon \frac{d \delta_{1}(k) k}{d k}+\varepsilon^{2} \frac{d \delta_{2}(k) k}{d k}+\cdots\right]\left(\varepsilon \delta_{1}+\varepsilon^{2} \delta_{2}+\cdots\right) k=\varepsilon^{2} \delta_{1} k \frac{d \delta_{1}(k) k}{d k}+\varepsilon^{3} \cdots \\
& \frac{d^{2} \vartheta}{d t^{2}}=\left[\varepsilon \frac{d \omega_{1}}{d k}+\varepsilon^{2} \frac{d \omega_{2}}{d k}+\cdots\right]\left(\varepsilon \delta_{1}+\varepsilon^{2} \delta_{2}+\cdots\right) k=\varepsilon^{2} \delta_{1} k \frac{d \omega_{1}}{d k}+\varepsilon^{3} \cdots \\
& \left(\frac{d k}{d t}\right)^{2}=\left[\varepsilon \delta_{1}(k)+\varepsilon^{2} \delta_{2}(k)+\cdots\right]^{2} k^{2}=\varepsilon^{2} \delta_{1}^{2} k^{2}+\varepsilon^{3} \cdots \\
& \frac{d k}{d t} \frac{d \vartheta}{d t}=\left[\varepsilon \delta_{1}(k)+\varepsilon^{2} \delta_{2}(k)+\cdots\right] k\left[\omega_{0}+\varepsilon \omega_{1}(k)+\varepsilon^{2} \omega_{2}(k)+\cdots\right] \\
& =\varepsilon \delta_{1} \omega_{0} k+\varepsilon^{2}\left(\delta_{2} \omega_{0} k+\delta_{1} \omega_{1} k\right) \cdots \\
& \left(\frac{d \vartheta}{d t}\right)^{2}=\omega_{0}^{2}+\varepsilon^{2} \omega_{0} \omega_{1}+\varepsilon^{2}\left(\omega_{1}^{2}+2 \omega_{0} \omega_{2}\right)+\cdots
\end{aligned}
$$

The substitution of Eqs. (A5), (A6), (A7), (A8), (A9) into Eq. (A4) yields:

$$
\begin{aligned}
& \frac{d^{2} T}{d t^{2}}+\omega_{0}{ }^{2} T=\varepsilon\left(-2 \omega_{0} \delta_{1} k \sin \vartheta-2 \omega_{0} k \omega_{1} \cos \vartheta+\omega_{0}{ }^{2} \frac{\partial^{2} T_{1}}{\partial \vartheta^{2}}+\omega_{0}{ }^{2} T_{1}\right) \\
& +\varepsilon^{2}\left[\left(\delta_{2} \frac{d \delta_{1} k}{d k}-\omega_{1}^{2}-2 \omega_{0} \omega_{2}\right) k \cos \vartheta-\left(2 \omega_{0} \delta_{2}+2 \omega_{1} \delta_{1}+\delta_{1} \frac{d \omega_{1}}{d k} k\right) k \sin \vartheta\right. \\
& \left.+\omega_{0}{ }^{2} T_{2}+\left(2 \omega_{0} \delta_{1} k \frac{\partial^{2} T_{1}}{\partial k \partial \vartheta}+2 \omega_{0} \omega_{1} \frac{\partial^{2} T_{1}}{\partial \vartheta^{2}}+\omega_{0}{ }^{2} \frac{\partial^{2} T_{2}}{\partial \vartheta^{2}}+\right)\right]
\end{aligned}
$$

The right parts of Eq. (23) can be written into the Taylor series expansion:

$$
\begin{aligned}
& \varepsilon f\left(T, T^{\prime}\right)=\varepsilon f\left(k \cos \vartheta,-k \omega_{0} \sin \vartheta\right)+\varepsilon^{2}\left[T_{1} f_{T}^{\prime}\left(k \cos \vartheta,-k \omega_{0} \sin \vartheta\right)\right. \\
& \quad+\left(\delta_{1} k \cos \vartheta-k \omega_{1} \sin \vartheta+\omega_{0} \frac{\partial T_{1}}{\partial \varphi}\right) f_{T^{\prime}}^{\prime}\left(k \cos \vartheta,-k \omega_{0} \sin \vartheta\right)+\varepsilon^{3} \cdots,
\end{aligned}
$$


where $f_{T}^{\prime}$ and $f_{T^{\prime}}^{\prime}$ is the first derivative of function $f\left(T, T^{\prime}\right)$ with respect to $T$ and $T^{\prime}$.

By combining Eqs. (A10) and (A11), and let the coefficients of the same order of $\varepsilon$ be equal, we obtain:

$\omega_{0}^{2}\left(\frac{\partial^{2} T_{1}}{\partial \vartheta^{2}}+T_{1}\right)=f_{0}(k, \vartheta)+2 \omega_{0} \delta_{1} k \sin \vartheta+2 \omega_{0} \omega_{1} k \cos \vartheta$

$\omega_{0}^{2}\left(\frac{\partial^{2} T_{2}}{\partial \vartheta^{2}}+T_{2}\right)=f_{1}(k, \vartheta)+2 \omega_{0} \delta_{2} k \sin \vartheta+2 \omega_{0} \omega_{2} k \cos \vartheta$

where:

$$
\begin{aligned}
& f_{0}(k, \vartheta)=f\left(k \cos \vartheta,-k \omega_{0} \sin \vartheta\right), \\
& f_{1}(k, \vartheta)=T_{1} f_{T}{ }^{\prime}(k, \vartheta)+\left(\delta_{1} k \cos \vartheta-k \omega_{1} \sin \vartheta+\omega_{0} \frac{\partial T_{1}}{\partial \vartheta}\right) f_{T^{\prime}}^{\prime}(k, \vartheta) \\
& +\left(\omega_{1}^{2}-\delta_{1} \frac{d\left(\delta_{1} k\right)}{d k}\right) k \cos \vartheta+\left(2 \omega_{1}+k \frac{d \omega_{1}}{d k}\right) \delta_{1} k \sin \vartheta \\
& -2 \omega_{0} \delta_{1} k \frac{\partial^{2} T_{1}}{\partial k \partial \vartheta}-2 \omega_{0} \omega_{1} \frac{\partial^{2} T_{1}}{\partial \vartheta^{2}}
\end{aligned}
$$

For $f(k, \vartheta)$ and $T_{1}$, were expanded into the Fourier series, i.e.:

$$
\begin{aligned}
& f_{0}(k, \vartheta)=g_{0}^{(0)}(k)+\sum_{n=1}^{\infty}\left[g_{n}^{(0)}(k) \cos n \vartheta+h_{0}^{(0)}(k) \sin n \vartheta\right] \\
& T_{1}(k, \vartheta)=v_{0}^{(0)}(k)+\sum_{n=1}^{\infty}\left[v_{n}^{(0)}(k) \cos n \vartheta+w_{0}^{(0)}(k) \sin n \vartheta\right]
\end{aligned}
$$

By substituting Eqs. (A16), (A17) into Eq. (A12), we obtain:

$$
\begin{aligned}
& \omega_{0}{ }^{2} v_{0}^{(0)}(k)+\sum_{n=1}^{\infty} \omega_{0}^{2}\left(1-n^{2}\right) v_{n}^{(0)}(k) \cos n \vartheta+w_{n}^{(0)}(k) \sin n \vartheta=g_{0}^{(0)}(k) \\
& +\left[g_{1}^{(0)}(k)+2 k \omega_{0} \omega_{1}\right] \cos \vartheta+\left[h_{1}^{(0)}(k)+2 \omega_{0} \delta_{1} k\right] \sin \vartheta \\
& +\sum_{n=2}^{\infty}\left[g_{n}^{(0)}(k) \cos n \vartheta+h_{n}^{(0)}(k) \sin n \vartheta\right] .
\end{aligned}
$$

Let the same coefficients of the Eq. (A18) be equal, one yields the following equations:

$$
\begin{aligned}
& g_{1}^{(0)}(k)+2 k \omega_{0} \omega_{1}=0, \quad h_{1}^{(0)}(k)+2 k \omega_{0} \delta_{1}=0, \\
& v_{0}{ }^{(0)}(k)=\frac{g_{0}^{(0)}(k)}{\omega_{0}^{2}}, \quad v_{n}^{(0)}(k)=\frac{g_{n}^{(0)}(k)}{\omega_{0}^{2}\left(1-n^{2}\right)^{\prime}} \\
& \omega_{n}^{(0)}(k)=\frac{h_{n}^{(0)}(k)}{\omega_{0}^{2}\left(1-n^{2}\right)}, \quad(n=2,3, \cdots), \\
& T_{1}(k, \vartheta)=\frac{g_{0}^{(0)}(k)}{\omega_{n}^{2}}+\frac{1}{\omega_{0}^{2}} \sum_{n=2}^{\infty} \frac{g_{n}^{(0)}(k) \cos n \vartheta+h_{n}^{(0)}(k) \sin n \vartheta}{1-n^{2}},
\end{aligned}
$$

From Eq. (A19), we know that: 
$\delta_{1}=-\frac{h_{1}^{(0)}(k)}{2 \omega_{0} k}=-\frac{1}{2 \pi \omega_{0} k} \int_{0}^{2 \pi} f\left(k \cos \vartheta,-k \omega_{0} \sin \vartheta\right) \sin \vartheta d \vartheta$,

$\omega_{1}=-\frac{g_{1}^{(0)}(k)}{2 \omega_{0} k}=-\frac{1}{2 \pi \omega_{0} k} \int_{0}^{2 \pi} f\left(k \cos \vartheta,-k \omega_{0} \sin \vartheta\right) \cos \vartheta d \vartheta$.

Similarly, using the same method, one obtains:

$f_{1}(k, \vartheta)=g_{0}^{(1)}(k)+\sum_{n=1}^{\infty}\left[g_{n}^{(1)}(k) \cos n \vartheta+h_{n}^{(1)}(k) \sin n \vartheta\right]$,

$T_{2}(k, \vartheta)=\frac{g_{0}^{(1)}(k)}{\omega_{0}{ }^{2}}+\frac{1}{\omega_{0}{ }^{2}} \sum_{n=2}^{\infty} \frac{g_{n}^{(1)}(k) \cos n \vartheta+h_{n}^{(1)}(k) \sin n \vartheta}{1-n^{2}}$,

$\delta_{2}=-\frac{h_{1}^{(1)}(k)}{2 \omega_{0} k}=-\frac{1}{2 \pi \omega_{0} k} \int_{0}^{2 \pi} f_{1}\left(k \cos \vartheta,-k \omega_{0} \sin \vartheta\right) \sin \vartheta d \vartheta$,

$\omega_{2}=-\frac{g_{1}^{(1)}(k)}{2 \omega_{0} k}=-\frac{1}{2 \pi \omega_{0} k} \int_{0}^{2 \pi} f_{1}\left(k \cos \vartheta,-k \omega_{0} \sin \vartheta\right) \cos \vartheta d \vartheta$.

From Eq. (A23) and Eq. (A24), we obtain:

$\delta_{1}=\frac{\zeta_{2}}{2}, \omega_{1}=-\frac{3 \zeta_{1} k^{2}}{8 \omega_{0}}$.

For Eq. (A22), letting $n=3$, we obtain:

$T_{1}=-\frac{\zeta_{1} k^{3}}{32 \omega_{0}^{2}} \cos 3 \vartheta$

Similarly, substituting $f_{1}(k, \vartheta)$ into Eq. (A27) and Eq. (A28), one yields:

$\delta_{2}=-\frac{3 \zeta_{1} \zeta_{2} k^{3}}{16 \omega_{0}{ }^{2}}, \quad \omega_{2}=\frac{3 \zeta_{1}^{2} k^{4}}{256 \omega_{0}{ }^{3}}-\frac{\zeta_{2}^{2}}{4 \omega_{0}}-\frac{9 \zeta_{1}^{2} k^{4}}{128 \omega_{0}{ }^{3}}$.

Substituting Eq. (A29) and Eq. (A31) into Eq. (30) and Eq. (31), one yields the following equations:

$\frac{d k}{d t}=\frac{\varepsilon \zeta_{2} k}{2}-\frac{3 \varepsilon^{2} \zeta_{1} \zeta_{2} k^{4}}{16 \omega_{0}^{2}}$,

$\frac{d \vartheta}{d t}=\omega_{0}-\frac{3 \varepsilon \zeta_{1} k^{2}}{8 \omega_{0}}+\frac{3 \varepsilon^{2} \zeta_{1}^{2} k^{4}}{256 \omega_{0}^{3}}-\frac{\varepsilon^{2} \zeta_{2}^{2}}{4 \omega_{0}}-\frac{9 \varepsilon^{2} \zeta_{1}^{2} k^{4}}{128 \omega_{0}^{3}}$.

For Eq. (A32), rounding off the small parameter high-order terms, we obtain

$k=k_{0} e^{\frac{\varepsilon \zeta_{2} t}{2}}$,

where $k_{0}$ is determined by initial conditions.

From Eq. (29), the non-linear vibration equivalent frequency can be obtained:

$\omega=\omega_{0}-\frac{3 \varepsilon \zeta_{1} k_{0}^{2} e^{\varepsilon \zeta_{2} t}}{8 \omega_{0}}-\frac{15 \varepsilon^{2} \zeta_{1}^{2} k_{0}^{4} e^{2 \varepsilon \zeta_{2} t}}{256 \omega_{0}^{3}}-\frac{\varepsilon^{2} \zeta_{2}^{2}}{4 \omega_{0}}$, 
$\vartheta=\left(\omega_{0}-\frac{3 \varepsilon \zeta_{1} k^{2}}{8 \omega_{0}}-\frac{15 \varepsilon^{2} \zeta_{1}^{2} k^{4}}{256 \omega_{0}^{3}}-\frac{\varepsilon^{2} \zeta_{2}^{2}}{4 \omega_{0}}\right) t$.

For Eq. (A22), letting $n=5$, we obtain:

$g_{0}{ }^{(1)}(k)=g_{2}{ }^{(1)}(k)=g_{4}{ }^{(1)}(k)=h_{2}{ }^{(1)}(k)=h_{3}{ }^{(1)}(k)=h_{4}{ }^{(1)}(k)=h_{5}{ }^{(1)}(k)=0$,
$g_{3}{ }^{(1)}(k)=-\frac{3 \zeta_{1}^{2} k^{5}}{64 \omega_{0}{ }^{2}}, \quad g_{5}{ }^{(1)}(k)=-\frac{3 \zeta_{1}^{2} k^{5}}{128 \omega_{0}{ }^{2}}$.

From Eqs. (A37), (A38) and (A26), one yields:

$T_{2}=\frac{3 \zeta_{1}^{2} k^{5}}{512 \omega_{0}^{4}} \cos 3 \vartheta+\frac{3 \zeta_{1}^{2} k^{5}}{3072 \omega_{0}^{4}} \cos 5 \vartheta$

Substituting Eq. (A30), Eq. (A39) into Eq. (27), one yields:

$T=k \cos \vartheta+\varepsilon T_{1}+\varepsilon^{2} T_{2}=k \cos \vartheta+\left(\frac{3 \varepsilon^{2} \zeta_{1}^{2} k^{5}}{512 \omega_{0}^{4}}-\frac{\varepsilon \zeta_{1} k^{3}}{32 \omega_{0}^{2}}\right) \cos 3 \vartheta+\frac{3 \varepsilon^{2} \zeta_{1}^{2} k^{5}}{3072 \omega_{0}^{4}} \cos 5 \vartheta$

From Eq. (14) and Eq. (A40), The displacement function of nonlinear vibration of a rectangular orthotropic thin film with damping is obtained. i.e.:

$$
\begin{aligned}
& w(x, y, t)=\sum_{m=1}^{\infty} \sum_{n=1}^{\infty} \sin \frac{m \pi x}{a} \cos \frac{n \pi y}{b} k \cos \vartheta \\
& +\left(\frac{3 \varepsilon^{2} \zeta_{1}^{2} k^{5}}{512 \omega_{0}^{4}}-\frac{\varepsilon \zeta_{1} k^{3}}{32 \omega_{0}^{2}}\right) \cos 3 \vartheta+\frac{3 \varepsilon^{2} \zeta_{1}^{2} k^{5}}{3072 \omega_{0}^{4}} \cos 5 \vartheta .
\end{aligned}
$$

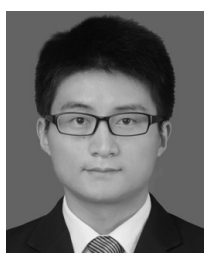

Xiaorui Fu is a postgraduate in machine design and theory at the Yanshan University, China. He received his Bachelorship in machine design and theory from Shandong Technology University in 2012. His primary research interests in the area of micro sensors.

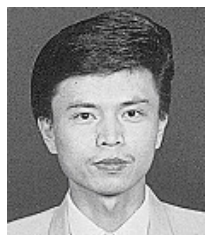

Lizhong $\mathbf{X u}$ is a full Professor in machine design and theory at the Yanshan University, China. He received his Ph.D. degree in machine design and theory from Yanshan University in 1999, and completed a 2-year postdoctoral fellowship in the first heavy machinery group of China from 2000 to 2002 . His primary research interests in the area of MEMS and electromechanical integrated system includes electromechanical coupled kinematics, statics and dynamics of the integrated system. 